\title{
Design and Analysis of Honeycomb Based Impact Attenuator
}

Praveen Malik, Petr Lepsik

Faculty of Mechanical Engineering, Technical University of Liberec, Studentska 2, 46117 Liberec 1, Czech Republic.Email: praveenmsrz@gmail.com, petr.lepsik@seznam.cz

The main idea of this research work is to understand the energy absorbing capacity of cellular solids when used in application of automobile as a safety measure to protect the occupants and structure of the vehicle. The full scaled model of the proposed design of the impact attenuator would be an expensive approach to follow. To understand the mechanism of energy absorption in honeycomb structure two types of experimental test were carried out. The first test includes a simple compression test of honeycomb structure only. The second experimental test consist of a composite sandwich of glass fiber and honeycomb under high speed drop test. Using the simple compression test the mechanical properties of the honeycomb were extracted in the out of plane behavior and then results from experimental data were calibrated with the FEM model. It was observed that very similar results were obtained in Experimental and FEM methods. And finally, the full scaled model analysis was carried in FEM package of ANSYS using mechanical properties of honeycomb extracted in simple compression test.

Keywords: Impact Attenuator, Compression, Energy Absorbing, Cellular Solids.

\section{Introduction}

The safety of the occupants in a vehicle can be compared to the objects which are packed inside a box for shipping from one place to another place at a distance. In this way the safety of the occupants depends on how much we spend on packaging i.e. on the safety of the occupants. The quality of protective packaging depends on the ability of it to convert kinetic energy into some other form of energy which is usually done from heat to plasticity, viscosity, viscos-elasticity or friction. This should be done keeping in mind that peek forces (and thus the deacceleration or acceleration) on the body should always be below the threshold as if its more than threshold it may cause injury to occupants of the vehicle [1]. Vehicle safety is one of automotive technology's most important fields of assessment. Automotive engineering is creating new methods to provide the occupants with passive and active safety systems and methods. Passive safety involves more extensive mitigation of the effects of accidents [2]. Passive Safety includes mitigation of accident consequences to greater extend. Passive safety measures can be done by a lot of different type of measures which include-

Inner-Safety- Inner safety can be done by making use of a seatbelt, belt force limiter, Seat belt travel limiter, airbag, side-bag, neck support etc. [3]

Outer-Safety- Outer safety is mainly used to keep the integrity of the vehicle during the collision which can be done by using body geometry, rigidity and deformation zone.
The driver's safety is most important when thinking in the safety of the vehicle. Secondary safety could be to safe guard the main structure of the vehicle during the crash scenario. To ensure safety of the driver and vehicle main structure there is possibility to use energy absorbing structure which can absorb bulk of energy which is generated during high speed collision of the vehicle[4]. The use of any absorbing structure will take up kinetic energy and would decrease the rate at which the vehicle deaccelerates during a high-speed crash scenario. These energy absorbing structures have been used in the automobile world and are commonly known as impact attenuator.

The impact attenuator[5] in simple definition can be defined as a device which can deaccelerate a vehicle in numerous steps to a complete stop or till the safety limits in which there would be no significant harm to the driver. By increasing the time in which the vehicle deaccelerates, the frame and driver can withstand the crash scenario without any significant harm to them. The bulk of impact energy generated during the crash is transferred into the deformation of the structure of the impact attenuator[6].

The impact attenuator can not only be integrated into vehicle structure but also on barriers on the roads to reduce the losses which can occur during a crash event.

The aim of this paper is to design and test an impact attenuator which would absorb significant energy during the crash event of vehicle and safeguard the occupants, structure of the vehicle. 


\section{Material Selection}

A lot of materials are available for honeycomb cores varying from Aluminum, Aramid Fiber, Stainless Steel, Thermoplastic and many more. The aluminum honeycomb cores were selected because aluminum would buckle more, and it would increase energy absorbing capacity of the structure. Also, aluminum honeycomb cores are more widely available in the market and it makes it less expensive to make a lowcost impact attenuator. The material selected for honeycomb was PCGA-XR2 3003 Aluminum Honeycomb with unit cell size of $6.4 \mathrm{~mm}$ by plascore.

\subsection{Compression Test of honeycomb Core-}

The out of plane behavior of honeycomb was tested using a universal testing machine by a simple compression test [7][8]. The honeycomb test piece consists of the dimensions $55^{*} 60^{*} 40.3 \mathrm{~mm}$. Similarly, 4 workpieces of identical dimensions were taken just to keep eye on the précised data obtained by the machine. The table below shows forces and deformation for different workpieces at the end time of compression test.

Tab. 1 End value of Force and Deformation w.r.t Time for Honeycomb.

\begin{tabular}{|c|c|c|c|}
\hline Work Piece No. & Time(s) & Force $(\mathrm{kN})$ & Deformation $(\mathrm{mm})$ \\
\hline 1st & 160.48 & 2.868 & 28.01 \\
\hline 2nd & 138.23 & 2.64 & 25.31 \\
\hline 3rd & 134.23 & 2.634 & 23.08 \\
\hline 4th & 131.23 & 2.55 & 22.83 \\
\hline Mean & & 2.67 & 24.81 \\
\hline Standard deviation & & 0.1363 & 2.408 \\
\hline
\end{tabular}

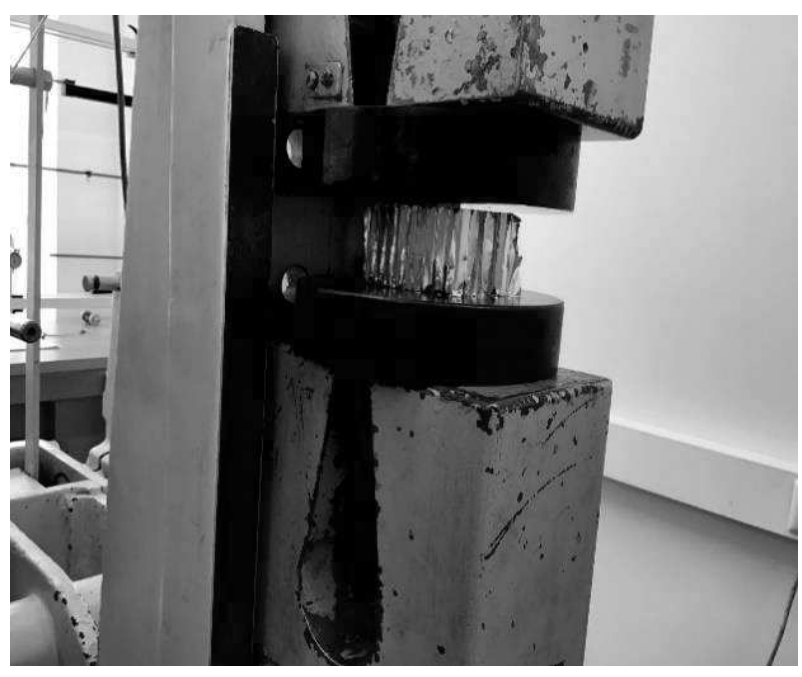

a)

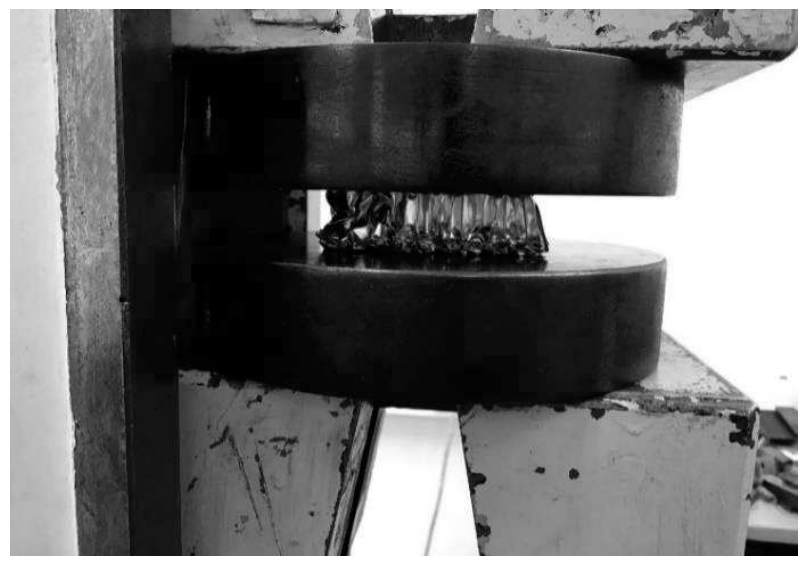

b)

Fig. 1 (a) Honeycomb core clamped for compression test. (b) Honeycomb core after compression test.
The force and deformation from the test was converted into the stress and strain as original length and area of the workpeicece was known. And from the stress and strain curve the stress strain was plotted.

Using LS-DYNA capabilities Displacement VS Force curve was imported which was obtained from the compression test of honeycomb core. Integration of this curve would lead to energy absorbed by the structure and the $\mathrm{Y}$ axis value will provide us with energy absorbed by our honeycomb core.

$$
\mathrm{W}=\int_{0}^{\varepsilon} \sigma(\varepsilon) d \varepsilon \quad \text { J] [9], }
$$

Where:

$\sigma . .$. Stress $\left[\mathrm{N} / \mathrm{mm}^{2}\right]$,

E...strain $[\mathrm{mm}]$.

\subsection{High Impact Testing}

The case in real would not be a simple compression when honeycomb compresses smoothly and absorbs energy rather it would be a case of high impact. So, to test the properties of honeycomb core in high impact scenario a high-speed penetration machine was used. The machine used for High impact testing of honeycomb structure was Coesfeld - High Speed Drop Tower[10].

The impact testing machine consist of a penetrator which comes from a height by the input velocity provided by the user. The speed is controlled using hydraulic pressure which helps to accelerate the penetrator by the speed provided by user. The penetrator consists of $5.3 \mathrm{~kg}$ weight and diameter of the penetrator $20 \mathrm{~mm}$. The speed of the penetrator was set to 11.238 $\mathrm{m} / \mathrm{s}$. In this case the dimension of the workpiece was $60 * 50 * 40.6 \mathrm{~mm}$ and a very thin layer of glass fiber of $2 \mathrm{~mm}$ was used so that the bigger part of the structure 
can be impacted by the penetrator as size of the penetrator was limited to $20 \mathrm{~mm}$ only. If honeycomb is used without sandwich of glass fiber or any other sandwich configuration, the honeycomb core would deform only in a local region of the penetrator, and it may also lead to just braking up of bonds of cell wall with neighboring cell wall. That's also one of the reasons why honeycomb when used for energy absorption application are always used as sandwich panels so that they can absorb much higher energies. The figure below shows the mounted honeycomb workpiece on the drop tower machine for high penetration test.

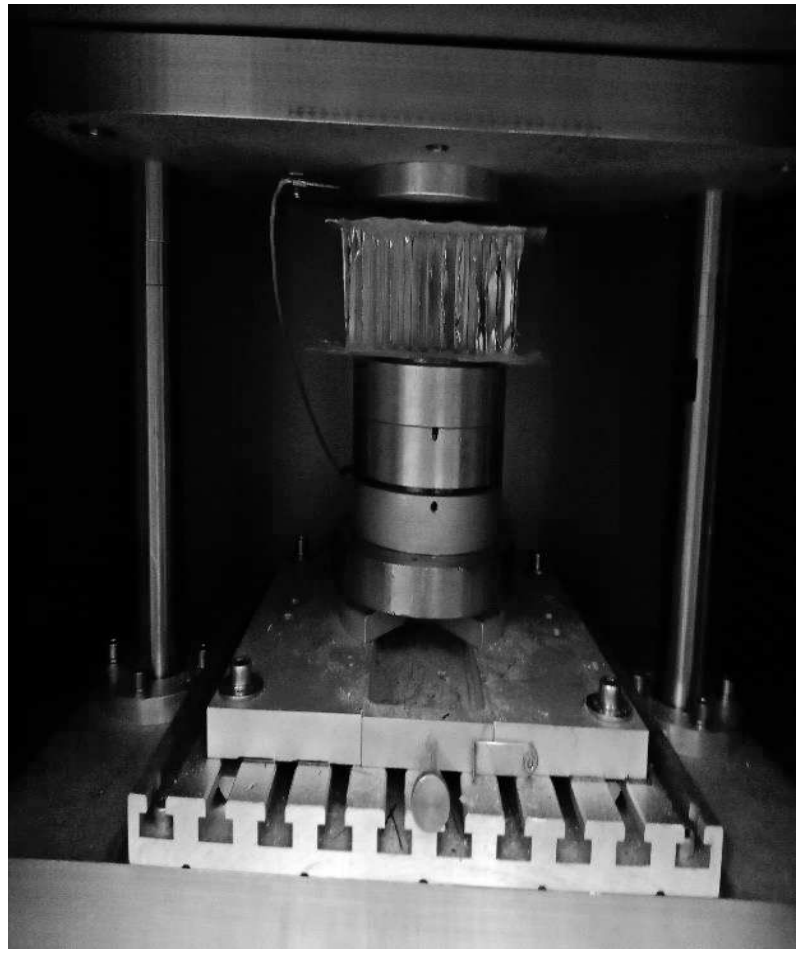

a)

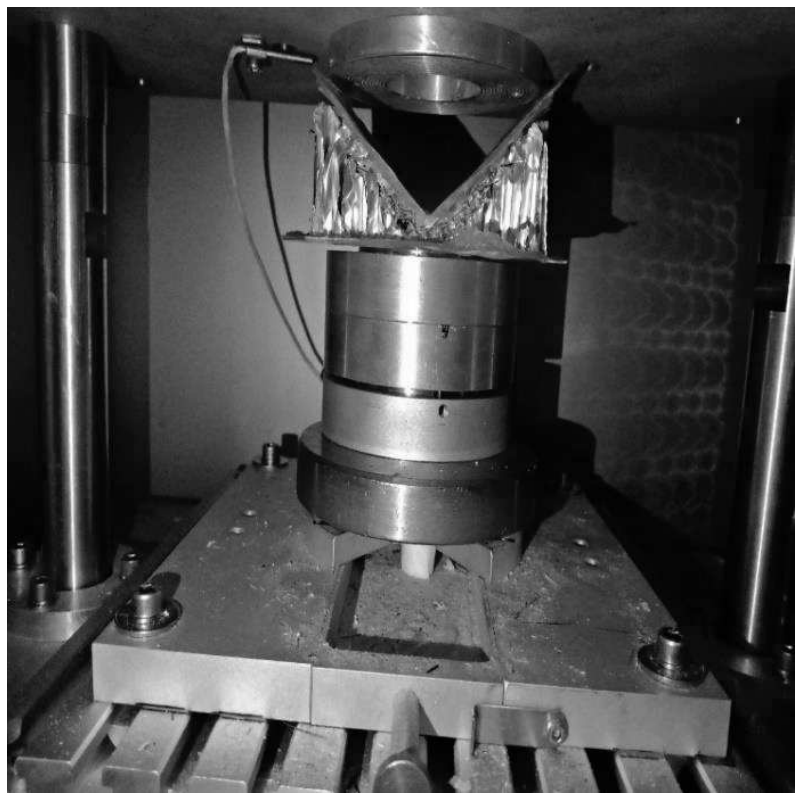

b)

Fig. 2 (a) Test work honeycomb workpiece before Test. (b) The Deformed Workpiece after test.

\section{Finite Element Analysis of Honeycomb Sandwich}

To model the honeycomb by exact dimension and then simulate it on FEM software is really a time-consuming process. The more efficient way thought was to just model block of same dimension as in the compression test and then apply properties of the material model which were obtained from compression test. So, in this way the model was made with the design modelling capabilities of SOLIDWORKS. The dimensions of the honeycomb core were $90 * 60 * 40.3$ $\mathrm{mm}$ with 2 plates on each side of $1 \mathrm{~mm}$ each. The Penetrator was modelled as a cylinder of $20 \mathrm{~mm}$ diameter. Sandwich block and penetrator were assembled in SOLIDWORKS with face mating. The file of modelled design was transferred from SOLIDWORKS to ANSYS using Parasolid format. In the Engineering Data of the ANSYS the general material model was taken, and it was given the Multilinear Isotropic Hardening. In multilinear isotropic Hardening the stress and strain curve generated in our compression test was provided.

Using the Space Claim the capabilities the shared mesh topology was used. The fine mesh was used to get the more precise results. The mesh consists of 55365 nodes and 5021 elements.

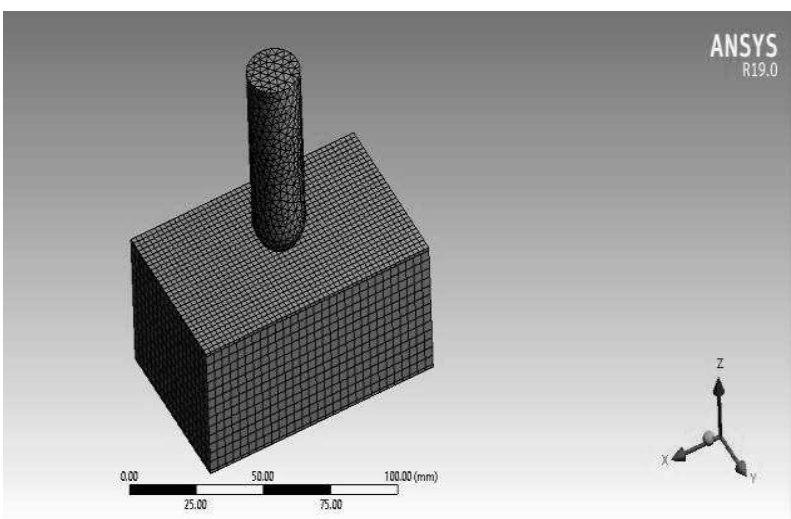

a)

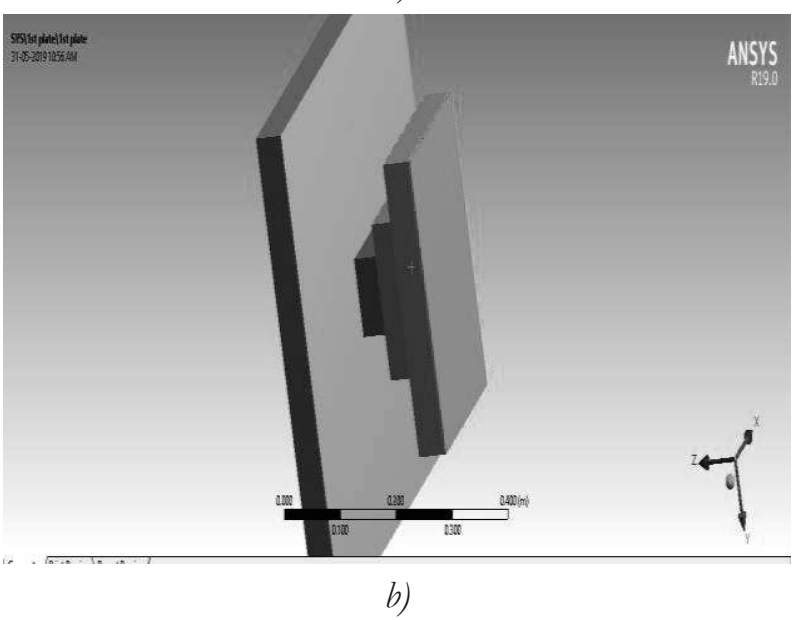

Fig. 3 (a) Meshed Honeycomb structure with penetrator. (b) Full-Scaled model of 3-layer honeycomb structure assembled with a wall. 
The penetrator was provided with a mass of $5.5 \mathrm{Kg}$ as it was in the high impact test. By varying the density in the material model of the penetrator this mass for penetrator was achieved. The penetrator was applied a velocity using component definition in global coordinate system. The applied velocity on the penetrator in the negative $Z$ direction was $11.5 \mathrm{~m} / \mathrm{s}$.

The fixture was applied at the rear plate of the sandwich honeycomb model and using face selection tool the fixture was applied. The fixed boundary condition made all translation and rotational degree of freedom to zero.

A 3-layer honeycomb Impact attenuator was Designed in SOLIDWORKS. The first layer sandwich consists of $180 * 300 * 50 \mathrm{~mm}$ dimensions. It is also supported by $1 \mathrm{~mm}$ thickness plates on both sides. The 2 nd layer consist of dimensions $120 * 160 * 50 \mathrm{~mm}$ and similar $1 \mathrm{~mm}$ plates on both sides and 3rd layer consist of $80 * 80 * 50 \mathrm{~mm}$ with $1 \mathrm{~mm}$ thickness plates on both sides. It was assembled with a modeled wall of dimension $500 * 500 * 50 \mathrm{~mm}$ using SOLIDWORKS assembly capabilities.

Now this assembled model was imported to ANSYS using a Parasolid file and same material model which we developed for our last scaled model was applied to the honeycomb composite. And a non-linear Concrete material model was applied to wall. The concrete material model is available as default material in engineering data of ANSYS. The effect of mass roll cage was applied by providing the mass of $300 \mathrm{~kg}$ to the most rear plate of the honeycomb structure from where it will be mounted on to the roll cage.

To get more accurate and précised results the fine mesh was generated. The more quality of mesh would help us to reduce errors from contact energy and hourglass energy losses. The rear end of the wall was fixed using the face selection tool. The rear plate of the honeycomb was provided with a velocity of 11.5 $\mathrm{m} / \mathrm{s}$ in $\mathrm{z}$-direction.

\section{Results}

The impactor of $20 \mathrm{~mm}$ diameter impacted on the honeycomb structure almost at the center and the structure looks to be deformed in a $\mathrm{v}$ shape as the area of the impactor was limited. The deformation occurred mostly in the region which was closer to the impactor. When looked closely the deformation in this case also occurred by buckling of tubes.

The energy vs displacement, energy vs time, Force vs time and force vs displacement graphs were obtained as the results of the output from the high impact penetration machine. The energy absorbed by the honeycomb structure was 340 joules. The energy Vs Displacement gap concludes that 340 joules energy was absorbed by displacement of $53 \mathrm{~mm}$ of the structure.

In the similar fashion the imported Displacement Vs Force graph was integrated using the LS-DYNA pre-post capabilities and figure below shows the integrated Displacement Vs Force and y axis value dictates to the value of the energy.

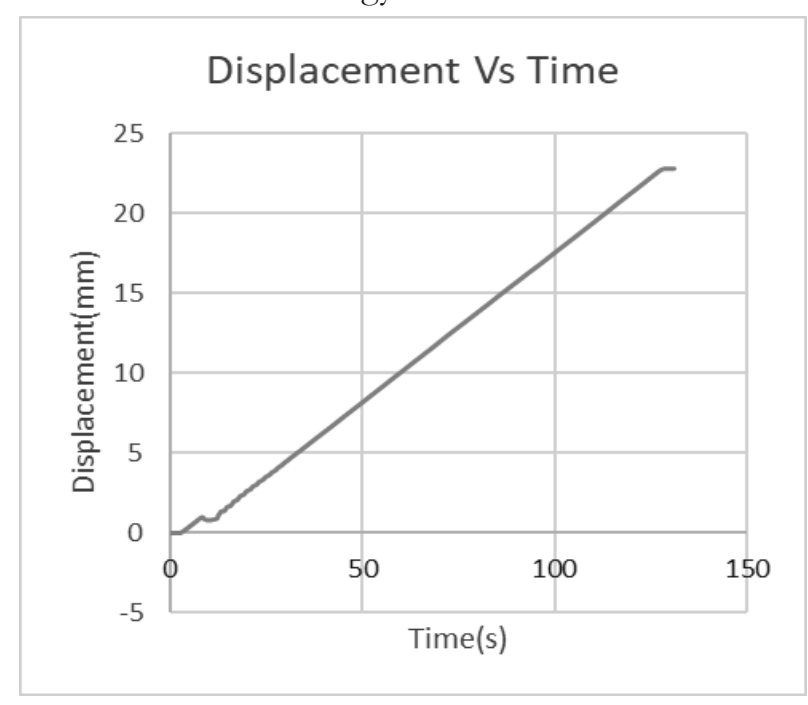

a)

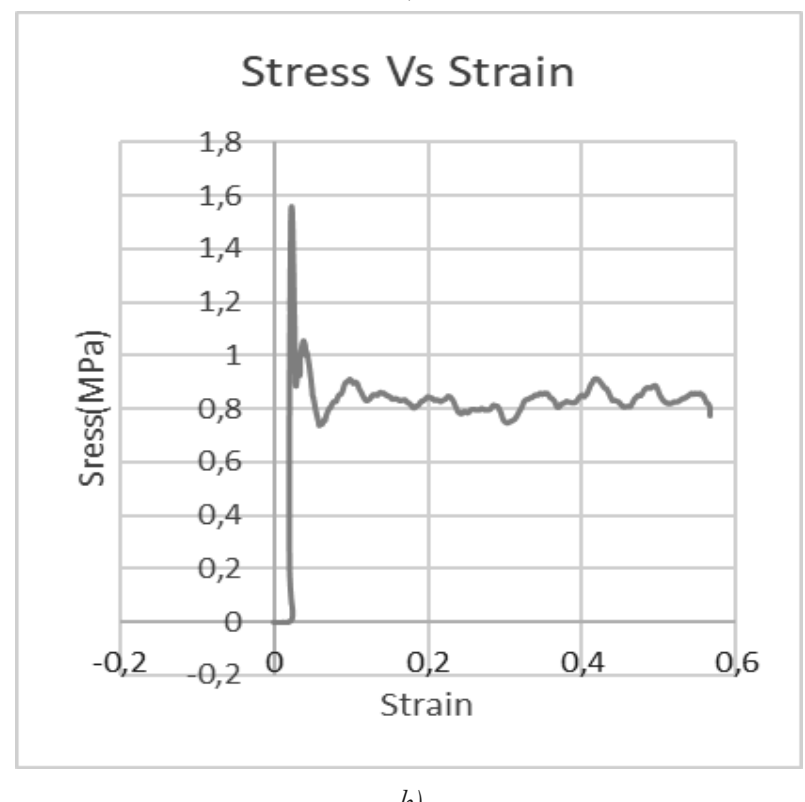

b)

Fig. 4. (a) Displacement Vs Time graph for Form structure. (b) Stress and Strain curve for Form Structure.

For comparison of honeycomb and foam work pieces out of different test the once which were with similar loading of forces were taken so that the results could be made comparable. It can be clearly noted that when honeycomb structure was compressed till 24 $\mathrm{mm}$ out of its $40.3 \mathrm{~mm}$ depth in out of plane behavior it absorbed an energy of 61.3 Joules. The energy absorbed by the honeycomb of almost same dimensions as of the foam was higher by a value of 13.3 Joules. Also, the honeycomb has lesser time duration in which it takes up loads and absorb energy. This makes honeycomb more suitable for our application as a more compact design would be able to absorb much more energy when compared to foam. 


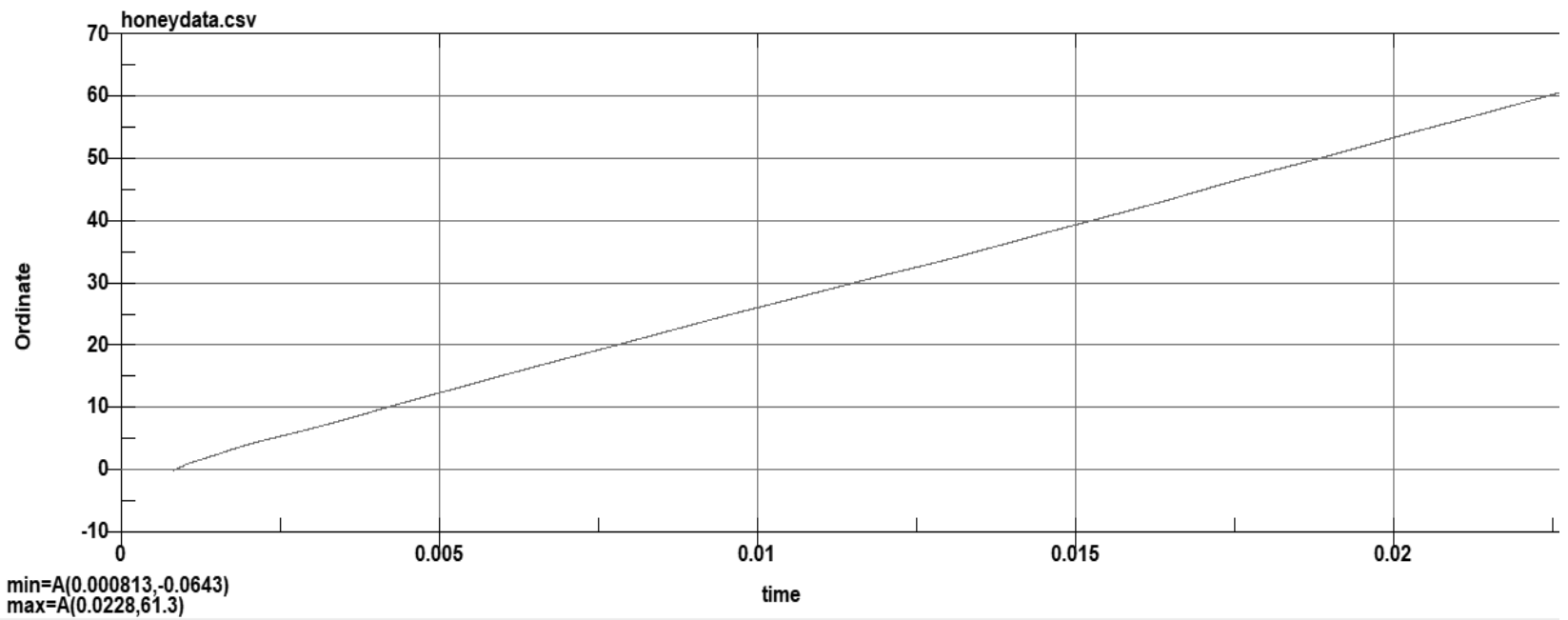

Fig. 5. (a) Imported Displacement Vs Force Data of Honeycomb Structure in LS-DYNA pre-post. (b) Energy vs Time for honeycomb.

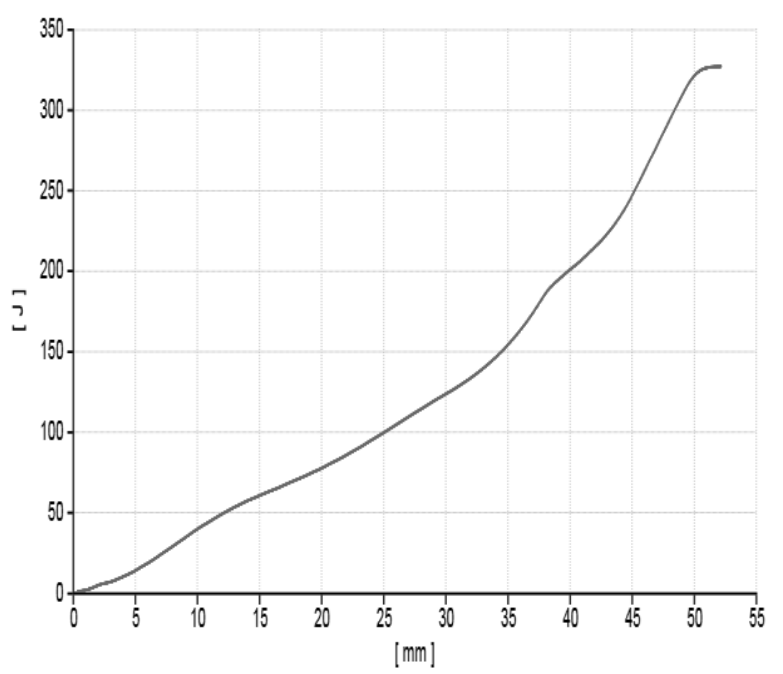

a)

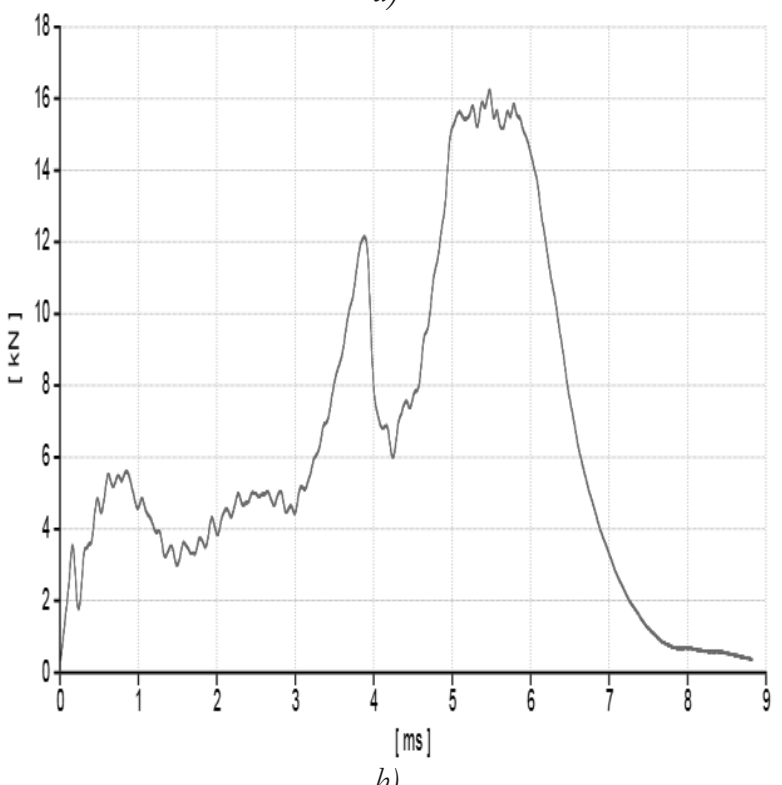

Fig. 6 (a) Energy Vs Displacement graph for Sandwich honeycomb in bigh penetation test. (b) Force Vs Time Graph for Sandwich honeycomb in bigh penetration test.
The Energy Vs Time graph concludes that 340 joules of energy was absorbed by the structure in 8.9 milliseconds. And Energy Vs Time graph is given below.

It can be clearly commented from our Force vs displacement graph that our structure of honeycomb takes a force of maximum $16.2 \mathrm{kN}$ by getting displaced by $53 \mathrm{~mm}$.

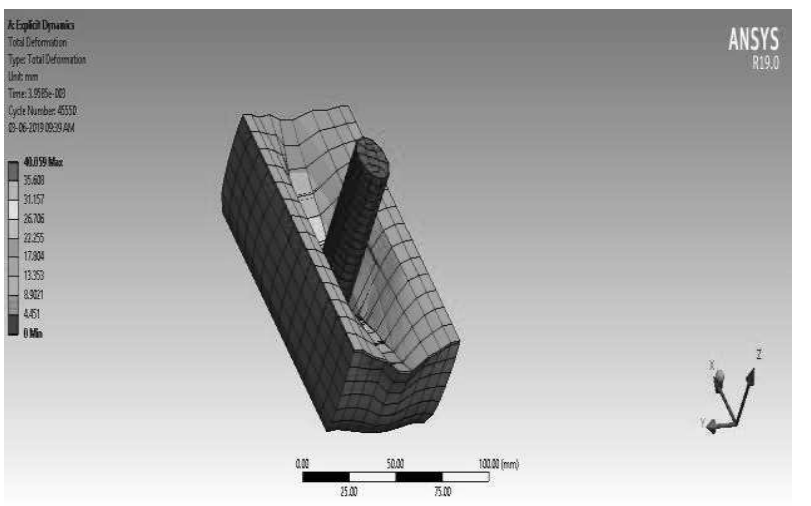

a)

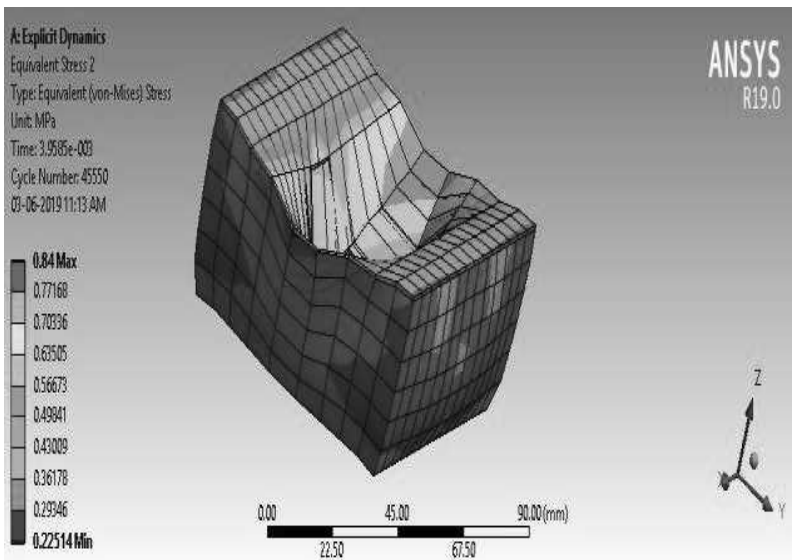

b)

Fig. 7. (a) Deformation in the Modelled Honeycomb structure. (b) Von mises stresses in the modelled honeycomb structure. 
The maximum of the Von-mises stresses occurred at the center of the structure where the penetrator made the strike. The value of the maximum Von-mises stresses was $0.84 \mathrm{MPa}$.

The most important parameter which we are interested in is the energy which the structure would be able to absorb and from the energy conservation we can conclude that the structure would be able absorb at least 300 Joules of energy. This value is similar to the energy value which we got in the high penetration test in real time. In the high penetration test experiment the structure absorbed an energy of 340 Joules. The figure below shows the energy summary of the model in ANSYS.

\section{Energy Summary}

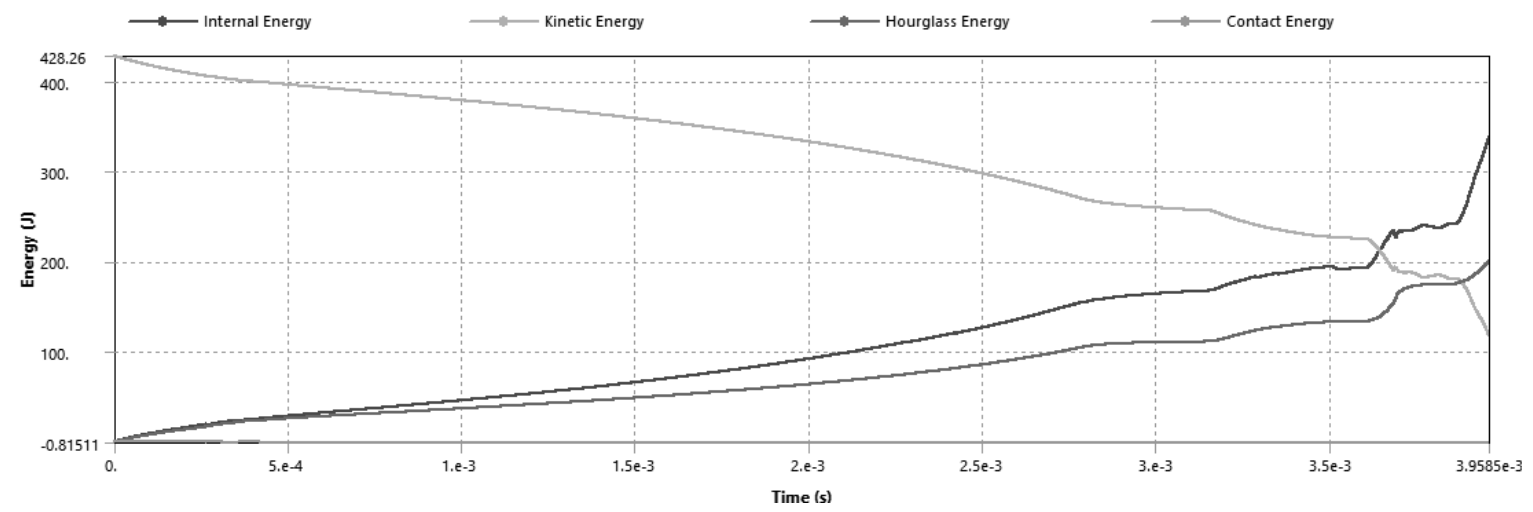

Fig. 8 Energy Summary of the Honeycomb model in ANSYS.

It can be said from force vs time graph that our honeycomb structure takes up a load of $16.2 \mathrm{kN}$ in 8.9 milli seconds. The energy absorbing capacity of honeycomb were increased significantly when it was used in a sandwich configuration with glass-fibre plates at the both ends. And it was observed that test workpeice would be able to absorb enegy of 340 Joules. The velocity was provided using component definition and the whole structure was compressed by a total deformation of $11.736 \mathrm{~mm}$.

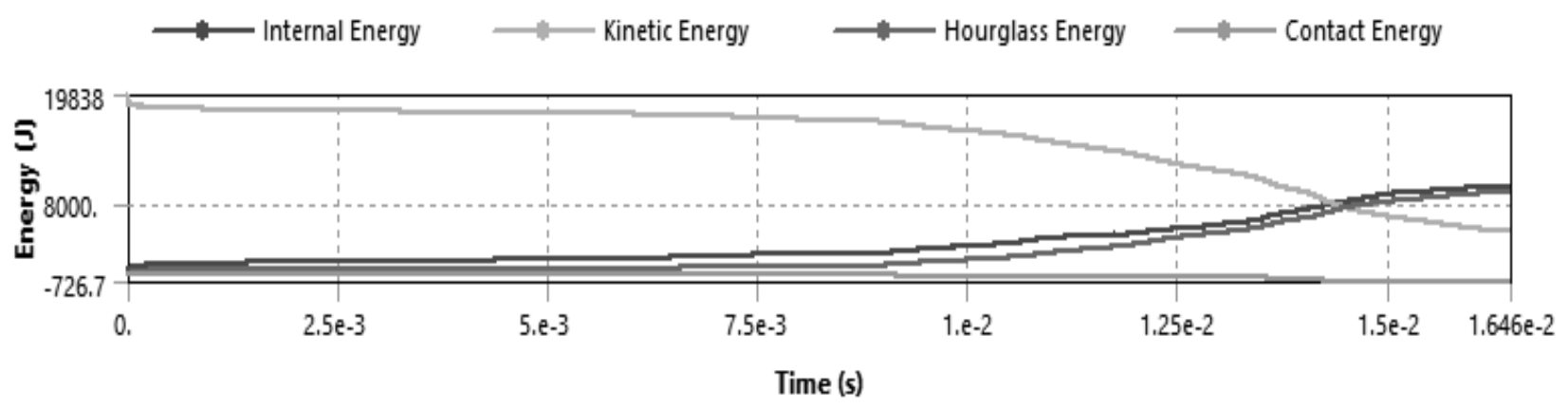

Fig. 9 Energy summary of the Full-scaled model of Impact attenuator.

The most use full result we are interested in is energy absorbed by the honeycomb structure. From the energy summary we can conclude that our structure would be able to take up energies up to 8000 Joules at least. There were less energy losses due to hourglass and contact as the material model used by us was highly non-linear and larger number contacts between 6 plates and 3 honeycomb structure.

\section{Conclusion}

- It can be concluded from compression test that when honeycomb specimen of $50 * 60 * 40.6 \mathrm{~mm}$ dimension is compressed can absorbed an energy of 61.3 Joules.

- The Snadwich comfiguragtion of honeycomb with any other material would significantly increase the energy carrying capacity of the structure and it was clearly proved in high speed impact test.

- The deformation of the sandwich structure of test piece happened in a $\mathrm{V}$-shape rather than uniformly because the small size of the penetrator as compared to workpiece. And this reason resulted in the more local failure around the impacting penetrator. 
- From FEM model of the Honeycomb almost similar results were obtained which matches with the experimental results and little deviation occured due to higly non-linear material properties of honeycomb and some assumptions which were made during FEM setup of model.

- The Full scaled model developed would be able to absorb energy up to 8000 Joules atleast. And by this way we can significantly reduce the damage that could occur to roll cage and occupant of the vehicle by a sufficient extend.

\section{Acknowledgement}

This work was supported by the Ministry of Education, Youth and Sports of the Czech Republic and the European Union - European Structural and Investment Funds in the frames of Operational Programme Research, Development and Education - project Hybrid Materials for Hierarchical Structures (HyHi, Reg. No. CZ.02.1.01/0.0/0.0/16_019/0000843).

\section{References}

[1] GIBSON, L. J., M. F. ASHBY, B. A. HARLEY (2010). Cellular Materials in Nature and Medicine, Cambridge University Press, 2010. ISBN: 9780521195447
[2] M. RAGURAMAN AND B. C. DAVID (2010). Lightweight impact crash attenuators for a small Formula SAE race car, International Journal of Crashworthiness, pp. 223-234, 2010

[3] Lecture notes, Automotive Engineering, Karlsruhe Institute of Technology (Germany).

[4] RAZ, K., HORA, J., PAVLATA, P. (2017). Unconventional Materials Usage in Design of Vehicle Bodies, Manufacturing Technology, 2017, 17(5):823-827

[5] Online material available on Impact Attenuator

[6] H. ZAREI, M. KROGER (2006). Optimum honeycomb filled crash absorber design, Germany: University of Hannover, Materials \& Design, pp. 193-204, 2006

[7] KUMAR DEVENDER, KHANNA NAMAN, Drop Test Analysis of Impact Attenuator for Formula SAE Car, International Journal of Scientific and Research Publications, Vol. 02 (10), pp. 01-04, 2012

[8] MÜLLER, M.,RUGGIERO, A., VALÁŠEK, P. (2017). Mechanical Characterisation of Metal/Polymeric Composite Waste/Metal Sandwich Panel, Manufacturing Technology, 2017, 17(4):530-536

[9] GIBSON, L. J., M. F. ASHBY (1997). Cellular Solids: Structure and Properties, 2nd ed. Cambridge University Press, 1997. ISBN: 9780521495608

[10] Coesfeld, Coesfeld Instrumented Impact Testers Manual 